\title{
Can I Reach You? Do I Need To? New Semantics in Security Policy Specification and Testing
}

\author{
Charalampos Katsis \\ Purdue University \\ West Lafayette, IN, USA \\ ckatsis@purdue.edu
}

\author{
Fabrizio Cicala \\ Purdue University \\ West Lafayette, IN, USA \\ fcicala@purdue.edu
}

\author{
Dan Thomsen \\ SIFT, LLC \\ Minneapolis, MN, USA \\ dthomsen@sift.net
}

\author{
Nathan Ringo \\ SIFT, LLC \\ Minneapolis, MN, USA \\ nringo@sift.net
}

\author{
Elisa Bertino \\ Purdue University \\ West Lafayette, IN, USA \\ bertino@purdue.edu
}

\begin{abstract}
The zero trust principle only allows authorized and authenticated actions in a computer network. A network policy satisfies the least privilege principle by minimizing the network permissions to only those needed by users and applications. However, administrators face many challenges in creating a least privilege policy since it requires a detailed understanding of the network topology and knowing the communication requirements of every network application and user. This paper addresses those challenges by introducing a graph-based policy specification framework to capture a network's communication requirements and a network compiler that turns those requirements into an enforceable policy. To offset the effort of building such a stringent policy, we incorporate patterns to spread the work of policy creation over time and people. In the paper, we first elaborate on how our framework's semantics enhances network security and resilience. We then introduce a Security Policy Regression Testing tool (SPRT), which leverages our framework's semantics, to test and reason about consistency, correctness, and relevance of network security policies. Finally, we outline relevant research directions.
\end{abstract}

\section{CCS CONCEPTS}

- Security and privacy $\rightarrow$ Access control; Authorization; Network security; Domain-specific security and privacy architectures.

\section{KEYWORDS}

Security policy enforcement; testing, access control; network security; zero trust; least privilege policy.

\section{ACM Reference Format:}

Charalampos Katsis, Fabrizio Cicala, Dan Thomsen, Nathan Ringo, and Elisa Bertino. 2021. Can I Reach You? Do I Need To? New Semantics in Security Policy Specification and Testing. In Proceedings of the 26th ACM Symposium on Access Control Models and Technologies (SACMAT '21), fune 16-18, 2021,
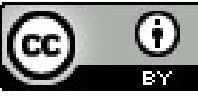

This work is licensed under a Creative Commons Attribution International 4.0 License.

SACMAT '21, June 16-18, 2021, Virtual Event, Spain.

(C) 2021 Copyright held by the owner/author(s).

ACM ISBN 978-1-4503-8365-3/21/06.

https://doi.org/10.1145/3450569.3463558
Virtual Event, Spain. ACM, New York, NY, USA, 10 pages. https://doi.org/10. $1145 / 3450569.3463558$

\section{INTRODUCTION}

Throughout the past decades, computer networks have grown to be large complex communication infrastructures [10,29], emphasizing the ability to communicate with everyone as opposed to only those you need to communicate with. Network functionalities include not only the communication expected by a user but many unexpected communications. For example, booking an Uber ride contacts at least eight different hosts on the Internet and network support functions [34].

Current network security relies on firewalls and middleboxes, acting as perimeter guards between subnets within the domain of interest or between a controlled domain and an uncontrolled network (e.g., the Internet) [2, 7, 29]. Administrators typically expend more energy creating stringent security policies on perimeter firewalls while deploying more flexible network policies to enable diverse traffic flows within the internal domain network [28, 29]. One reason is that network flexibility enhances communication efficiency and minimizes the risk of application failures due to excessively restrictive policies. Also, the organization's threat model may not assume adversarial presence within the organization, thus blindly trusting all the traffic that flows within the network. Finally, configuring well-tailored policies for the internal network requires a deep understanding of the traffic that should be allowed in all the network segments, and researchers have found manual tightening of policies to be significantly error-prone $[2,7,11,15]$. However, once attackers breach the perimeter, flexible, permissive policies allow attackers to move both laterally and deeper into the network.

Recent approaches to prevent attackers' lateral movements and to enhance network security apply the concept of zero trust architecture (ZTA) [28, 29]. ZTA has been developed on the premise that only authorized and authenticated actions may be executed in a network as this architecture assumes an adversarial presence. In other words, no trust between any entities may be assumed unless explicitly specified.

A ZTA-based network aims to control access to resources, such as applications or data (e.g., a database), by entities (users or assets) needing access to them. One way to support fine-grained access control is through micro-segmentation by which a network device (e.g., gateway, router, switch, firewall) acts as a policy enforcement 
point (PEP) protecting a resource or a group of resources. PEPs use rules to enforce a common level of trust for allowable traffic. For example, a PEP on the path from a source to a destination allows traffic to pass only if the source falls into a legitimate range of IP addresses, while a subsequent PEP in the path allows traffic to pass only if specific protocols (e.g., ftps) are used. By minimizing permissions to what is absolutely necessary to ensure network operations, we can avoid unnecessary permissions and enforce a least privilege policy.

However, busy network administrators find detailed permission management a daunting and time-consuming task [34]. First, the communication requirements of assets (e.g., services, devices, virtual and cloud components, applications, infrastructure components) and users need to be well understood. Such requirements include details about all the necessary communications for each network entity. For example, an asset might need to communicate with an online service through the HTTPS protocol to synchronize its clock, or a user might need access to a particular resource to perform a specific task. Second, administrators need to formulate a network access control policy specifying which users may interact with which assets and how. Finally, administrators need to translate the policy into rules and deploy them on the appropriate PEPs on the network, using different concepts based on the network topology like network masks. Capturing and sharing policy patterns can reduce this effort, just like capturing an algorithm in a software library reduces effort. By defining patterns in terms of meaningful concepts like roles and sets of resources, the overall effort can be reduced [32, 33].

Application developers have a detailed knowledge of application requirements. However, it needs to be in a form that will aid network administrators in authoring the network access control policies. Furthermore, developers could specify abstract roles and tasks and how those interact with their applications based on use cases. Subsequently, administrators could extend the developer-specified communication requirements, roles, and tasks to author the specific network access control policy that meets local needs. Hence, such a better understanding of the network results in enhanced network security and a significantly lower burden for network administrators.

This paper proposes a graph-based policy specification framework to capture the semantics mentioned above and provide a more coherent understanding of the network and its requirements. Our framework divides the policy specification into two parts: (1) the static policy, which includes application communication requirements as well as the high-level role-based access control model, and (2) the local policy, which composes of an access control model and local network configurations. The static policy may be authored by the application developers and extended by the domain administrators based on their domain policies and needs.

Accurately translating static and local policies into rules enforced in the underlying domain network is fundamental for successfully building a ZTA and enforcing a least privilege policy. As manual policy translation is generally not scalable and is a significantly error-prone process $[2,3,7,11,15]$, our prior work [34] has proposed the idea of a network-wide security policy compiler. The compiler receives the static and local policy specifications as input,

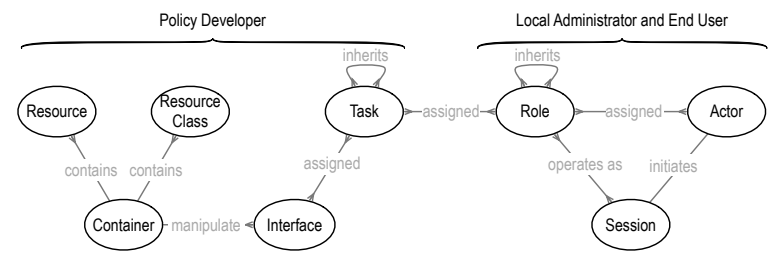

Figure 1: Separating common patterns of security policy specification can reduce the workload of the local administrator. Here, application developers define tasks and a task hierarchy that the local system administrator can incorporate into their local policies.

compiles different sets of rules, and finally deploys them on the appropriate PEPs.

Such an approach produces an additional benefit. By precisely specifying the communication requirements, it becomes possible to perform systematic testing to guarantee that the deployed network policy meets all the requirements. Testing reveals whether or not the deployed network policies enable applications to perform their missions successfully while ensuring the least privilege policy. Moreover, it allows checking the ruleset correctness and relevancy on every network device. Hence, in this paper, we propose a Security Policy Regression Testing (SPRT) tool to automate network-aware policy analysis and verification and perform systematic policy enforcement testing. SPRT receives both static and local policy specifications, as well as the local network topology. By analyzing information flows allowed by the policy, it can show the communication requirements are satisfied and if the least privilege policy is enforced.

In addition to the testing capabilities, SPRT supports policy change impact analysis using the semantics captured in the graphbased policy specification framework. As the complexity of networks increases, tools for the impact analysis of policy changes are critical as the impact is often unclear even to the administrators performing the changes $[14,18]$, and these changes may cause unintended failures. For example, an administrator can alter the existing policies by retracting rarely used permissions from the network [34]. In this case, SPRT aims to assess whether the impact of such a policy action may cause unintended failures and identify the service that will fail.

The rest of the paper is organized as follows. Section 2 introduces the idea of considering policies as reusable patterns for network security policies. Section 3 provides details about our graph-based policy specifications. Section 4 outlines our approach for security policy regression and testing. Section 5 introduces open research directions related to specification and optimal deployment of network security policies and discusses extensions and applications of our framework. Section 6 concludes the paper.

\section{POLICY PATTERNS AS SOFTWARE}

This work started as we looked at how to apply software engineering techniques to developing network policies. Software for complex projects allows the hard work of writing code to be split up between library and component developers and the coder customizing the functionality. Here we wanted to look what are the patterns 
in network security policy that we can discover and capture so that others can easily reuse those patterns.

We started with the JASPER model $[20,34]$ that splits role creation into roles created by the local system administrator and tasks created by the policy pattern engineers. Software developers would develop a set of tasks for their application that address how they envision the system being used. Subsequently, the local system administrator would customize these policy starting points for their environment. If software developers specify well-engineered tasks, the local administrators can simply assign them to roles with no role engineering. JASPER differs from the NIST RBAC model [13] in that it organizes resources into containers which can only be reached through the containers interfaces (see Figure 1). This difference allows us to create patterns of resources like the users' home directories rather than explicitly listing all the resources. We apply this same pattern in network policies to define abstract patterns like "user laptops" so we do not have to define each laptop at pattern development time.

\section{POLICY SPECIFICATION VIA GRAPHS}

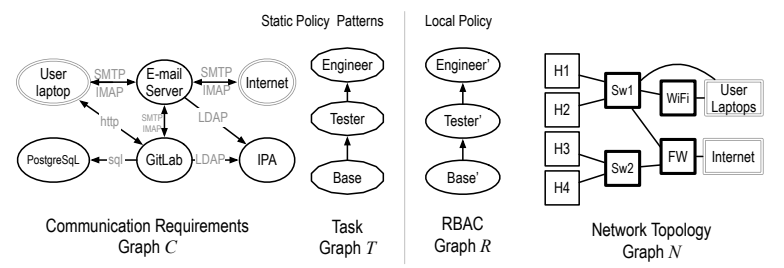

Figure 2: The base graphs used by the network compiler, separated into static design information and local configuration graphs.

Graphs provide a flexible abstraction to model and reason about both role-based policies and network topology needed by the network compiler. We divide the entire policy into seven graphs: two capturing requirements and common security patterns, two capturing local policies, two mapping graphs, and one graph capturing network protocol communication sequences. The RBAC graph provides the familiar object-oriented abstraction of permissions. The task graph duplicates the functionality of the RBAC graph to separate cross-organization patterns $[32,33]$ from local policy requirements. The task graph associates permissions to roles using the JASPER model of containers and container interfaces. Here the permissions correspond to allowing communications to pass through a PEP. The network topology graph describes the physical network topology of hosts, servers, switches, routers, and firewalls. Our novel communication requirements graph captures the communication requirements patterns between different network services. The separation into multiple graphs divides the work of creating a security policy between multiple individuals, from creating static security patterns for commonly integrated software to local security requirements. In particular, the decomposition of the framework into multiple graphs allows for the description of security patterns independently of network topology and local network constraints.

\subsection{Communication Requirements Graph $C$}

The communication requirements graph $C$ captures the abstract representation of network communication requirements independent of the network topology. These requirements become critical for testing the resulting policy for correctness to ensure that only required communications are allowed. The requirements graph captures the dependencies between services, utterly devoid of network topology constraints. It includes application-specific connections like E-mail servers connecting to IPA servers via LDAP for authentication and low-level requirements like host connections to DNS servers.

We label the edges of the $C$ graph with the type of communication, for example, $A \stackrel{h t t p}{\longrightarrow} B$ so that that the network devices can control the proper ports. Local designs can remap ports, which the network compiler must address.

Strict network policies require knowing who has permission to initiate the communication, resulting in a directed graph. In the Figure 2, the nodes fall into two categories: classes like "User Laptops" and the Internet, and specific instances like an E-mail server. It is important to stress that, in the requirements graph, the E-mail server represents a pattern of access to the server. It is not instantiated until the network compiler combines it with the network topology and maps it to a specific host.

\subsection{Role-Based Access Control (RBAC) Graph $R$ and Tasks $T$}

The RBAC graph groups permissions into an object-oriented design to capture common access patterns, and the local administrator defines it. This resembles the familiar RBAC policy in security literature. However, rather than mapping roles to permissions, it maps roles to tasks from the task $(T)$ graph. The task graph parallels the familiar RBAC graph but is separated to represent the different roles in creating the security policy. Application developers organize the permissions of their applications into tasks to save effort for the local administrators. We use the JASPER model [20, 34] that defines permissions as access to resources through a container interface as shown in Figure 1. This allows us to pragmatically define resources, such as a user's private laptop or files in their home directory, without explicitly listing them.

The communication requirements graph defines a set of network resources available to assign to users in the task graph. The model considers the network as a container, with the network services being resources inside the container. Resource patterns like "User laptop" have their own interface that instantiates one laptop per user assigned to that resource. The policy is defined such that only the owner of the laptop has access to it.

\subsection{Network Topology Graph $N$}

We represent the topology graph as an undirected graph. Nodes correspond to hosts and network Policy Enforcement Points (PEP) like switches, routers, and firewalls. The model types each PEP because different types have different capabilities to enforce network policy that the network compiler must consider. For example, an inexpensive PEP may only support a small number of filter rules. The topology graph represents the physical layout of the network 
that cannot be changed by software means. For the network compiler it represents the basic elements available for network policy enforcement.

In our approach, the network topology only defines the portion of the network under the local administrators' control. A firewall usually marks the boundary of the topology graph, and while local administrators cannot change the policy beyond the firewall, they can change the policy on the firewall, which may limit what hosts on external networks can access within the controlled network.

Even the topology graph contains patterns. We have created one special class of network for the Internet since, of course, most policies will refer to it. Another pattern is "User Laptops", which represents a dynamic number of hosts based on the number of users and where they connect to the network. For example, rather than enumerate all user laptops, they are represented by the pattern of allowing them to connect to the network via a WiFi router or a physical router.

\subsection{Mapping Graphs: User $U$ and Host $H$}

Local administrators assign roles to users, which the model captures in the user graph $U$. Local administrators also decide which network services to run on which host, which we capture in the host graph $H$. The $H$ graph allows for multiple services to run on a single host or to distribute a network service over a cluster of hosts for performance. The $H$ graph also defines the user laptops connection points to the network, for example, two different WiFi routers on different floors. Both the $U$ and $H$ graphs represent more dynamic aspects of the policy as users change roles or administrators migrate services to new hardware.

\subsection{Protocol Sequence Graph $P$}

At an abstract level, the communication requirements graph provides concise insight into the communication needs of network services. However, the realities of actual network protocol add an extra layer of complication. Many protocols are uniform, but some use asymmetric paths that impact security policy on network devices. For example, connections to an FTP server are initiated on one port, but file transfer happens on another port. This graph captures the communication sequence to ensure the policy captures all necessary ports.

\subsection{Creating the Policy}

The network compiler combines all of these graphs to create the policy for the network. If services or user laptops migrate, the network policy must be recompiled to ensure that policy satisfies the new conditions. All the necessary information may be calculated by traversing the graph. For example, combining the communications, task, RBAC, and user graphs $(C \times T \times R \times U)$ allows one to determine the number of individual user laptops since it instantiates a laptop for each user assigned to a role that has access to the "User Laptop" resource pattern from the communications graph.

Other properties can be derived from the graphs. For example, the communication requirements graph captures the constraints of what communications the network must allow. By inverting that graph, the network compiler builds a set of constraints for denied communication paths (see Figure 3).

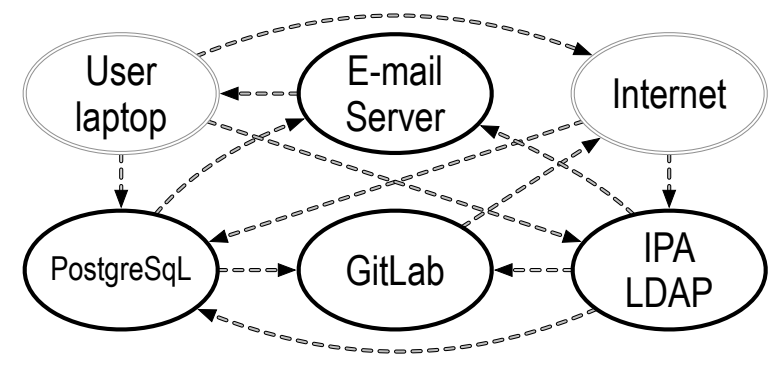

Figure 3: The network compiler can infer the set of communications that are not required and should be denied by inverting the communication requirements graph $C$ of the security policy.

Graphs provide an ideal model of network traffic. The complexities of a real network, like performance, multiple network paths, and packet ordering, make the network compiler's job more challenging. Large, complicated networks can provide multiple paths between two communicating hosts. Administrators may have designed the network for redundancy or performance. While the network compiler could remove redundant paths, such changes may drastically impact the performance of the local network. Currently, we have the compiler note the redundant paths but not remove them. Most wide-area networks have multiple paths, but the local administrators do not control them, and thus they have no impact on the network compiler.

Network protocols do not guarantee packet order. The low-level network software on the receiving host assembles the packets into the proper order. In normal wide-open networks, packet order does not matter because all the packets have permission to flow. In our model, it can impact how to enforce the protocol sequence graph $P$. Rather than model all the actual packets that flow in sequence, graph $P$ models only the events that must happen in sequence. For example, the client must initiate the connection to a web server first. This approximates the expected packet order sequence.

Network administrators typically configure the network to meet local performance goals. For example, servers that communicate frequently may be placed on the same network segment. However, most networks evolve over time, changing configurations until achieving acceptable performance. Eventually, a network compiler could observe network traffic and suggest a change to the physical configuration to improve performance. However, for our initial designs, we assume that the network compiler cannot change the physical network $N$ or the mapping of services to hosts graph $H$. If administrators migrate services or change the network topology, the policy must be recompiled.

\subsection{Comparing the Dynamic Nature of Security Enforcement Policy}

The set of graphs allow us to express a variety of security patterns that vary in policy complexity. The communication requirements graph represents relatively static policies defined by how the network servers interact and is best defined by the application developers. Standard network protocols, which are extremely static, are defined by the network protocol graph and best defined by network protocol developers. 
Local administrators define their own network topology and determine how to satisfy their local security requirements with the security policy building blocks supplied by the application developers. Local administrators instantiate the security patterns defined by others, or if necessary, create their own patterns. Even then, the dynamic nature of networks requires the policy to adjust to changing conditions, like users moving their wireless connection from a conference room back to their office. Such an approach allows security enforcement patterns to be validated and shared by experts. We believe this approach minimizes the work for local system administrators while giving them maximum flexibility. A graph-based approach also defines a clean set of required communication paths and paths that can be denied without impacting application behavior. This allows one to create tools for security policy regression testing and reduce the network's attack surface by reducing network permissions without impacting applications.

\section{THE SECURITY POLICY REGRESSION TOOL}

Ensuring enforcement policy correctness minimizes application and user communication failures while supporting claims of a least privilege policy. The Security Policy Regression Testing (SPRT) tool tests the policies generated by the network administrator or network compiler against mission-critical requirements. Regression testing the security policy detects unnecessary or inconsistent policies before their deployment.

SPRT must examine all the possible paths between any communicating endpoints to ensure that the generated rules correctly reflect the communication requirements and detect potential discrepancies in the network topology, such as a path that bypasses policy enforcement points or communications that do not reflect the communication requirements. Such discrepancies may compromise the effectiveness of the PEPs and undermine the network's security.

Inconsistencies often arise due to access control (AC) policy modifications. For instance, an organization introducing new network services must import the tasks associated with the service and modify the role policy to assign access to users. An organization could also modify its network infrastructure by introducing additional resources, thus requiring adjusting the network policies. Every alteration of the environment impacts the network's effectiveness, and it is possibly subtle and highly complicated to detect through manual investigation. For example, adding a new web interface results in adding a new resource to a container, impacting the task, role definitions, and ultimately what a user can access. If we change access permissions to a container interface, we need to determine what tasks and roles are affected by the change and for which set of resources. An analysis tool that automatically determines the impact of changes to the AC policies can significantly facilitate network administrators' tasks [14]. Hence, we also integrate such AC policy change-impact analysis into SPRT to better understand how changes impact network tasks operation.

\subsection{Network-aware Policy Verification}

SPRT performs regression testing by creating a set of proto-packets that correspond to all the network packets that must flow through the network to satisfy the communication requirements graph $C$.
Proto-packets only contain source and destination addresses and network protocol. SPRT tests if the proto-packets can flow on a graph-based model of the network. If all simulated proto-packets reach their destination successfully, it proves that the policy will not hinder applications and user activities. If the policy does not allow a proto-packet through, SPRT reports not only the details about the proto-packet, but, also, it maps the proto-packet back to the edge in the communication requirements graph that was not satisfied.

Moreover, to make claims of least privilege, SPRT must also test the inverse of the communications graph, which we call denied graph (see the example in Figure 3). SPRT again uses the concept of proto-packets sent through the network model to test the denied communications. However, this time, to ensure a least privilege policy, none of the packets must reach their destination.

SPRT also tracks the set of rules triggered while testing the protopackets generated based on both the communication graph and the denied graph. Any rule that was not used has no impact on the policy and can be safely removed, improving performance and reducing policy complexity.

Therefore, SPRT needs to test the communication requirements given the PEPs' rule sets and, also, to reason about the correctness of those rule sets. Hence, the tool aims to achieve the following four objectives.

Objective 1 - Communication requirements testing. To test the reachability of entities that must communicate via the specified protocol as per the $C$ graph, proto-packets must successfully traverse the network for all possible routing paths from a source to a destination specified in the $C$ graph. Note that the administrators may configure the network with multiple paths to increase bandwidth and reliability [35]. Therefore all the paths need to be examined in order to guarantee reachability.

Unsuccessful proto-packet transmissions reflect a policy that blocks communications that are supposed to be permitted. Thus, such an approach allows administrators to pinpoint the faulty protopackets and analyze the incorrect rules. In particular, the analysis reveals:

(1) Information about the proto-packet under testing, which is generated based on the communication requirements $(C)$ and the protocol sequence $(P)$ graphs.

(2) The network nodes and the respective security rules preventing the proto-packets from being transmitted. Note that there could be a rule that drops the packet on a particular node, or the packet could be dropped because there is no matching rule (i.e., the default deny case).

(3) The network paths the packet has traversed.

(4) The host machines and users or services for which the packet transmission is tested. We express this mapping through the graph $H$.

(5) The tasks or patterns that fail based on the local policy graphs $R$ and $U$.

(6) The communication requirements that are not meet.

Objective 2 - Least privilege communications. SPRT must also test the PEPs' configuration to ensure a least privilege policy by testing whether unintended communications can be performed. In other words, find where there is a path for any two entities to 
communicate, given that no such requirement exists in the $C$ graph. If no such path exists, we can conclude that the least privilege policy in communications is enforced. We can test the feasibility of unintended communications by computing the denied graph $\left(C^{\prime}\right)$ of the communication requirements graph $C$, which represents communications that must be denied. Any two vertices are adjacent in $C^{\prime}$ only if they are not adjacent in $C$. Thus, we can employ proto-packets representing communication requirements in $C^{\prime}$ and test whether they successfully reach their destination, which was supposedly denied in the original communication requirements graph $C$.

Objective 3 - PEP bypass. SPRT must test whether one or more PEPs are bypassed. Typically, routers forward packets based on path metrics (determined by protocols such as OSFP [25] or EIGRP [31]) or routing policies, which can lead to multiple paths. In the case of multiple paths, it is vital to check the rulesets' consistency across the PEPs present in those paths. For instance, consider a rule placed on a PEP $k$ that prevents a particular sub-network of users from accessing a service. Then, consider a second path between that sub-network and the service that is not passing through PEP $k$ Such a second path bypasses $k$ and allows the traffic to flow despite the rule being enforced on a PEP. Therefore, SPRT must discover paths that bypass the network PEPs.

Objective 4 - Reasoning about the deployed rulesets. SPRT must disclose its findings for administrators to evaluate and adapt the rulesets deployed on each PEP. SPRT identifies the relationship between tasks and network traffic, namely, which task is responsible for generating which portion of the traffic on the network. It also determines what traffic passes through any node. Thus, by comparing the identified traffic with the communication requirements graph, SPRT can detect traffic that should not flow through the network and suggest rules to prevent it. However, designing an intelligent mechanism for rule placement is challenging, as discussed in Section 5. Additionally, by comparing the identified traffic with the deployed security rules, SPRT can detect which rules allow illegitimate traffic to pass, and also it can reveal irrelevant rules (i.e., rules not triggered during testing). The latter type of rules can be safely eliminated without impacting the network and its requirements.

\subsection{Policy-change Impact Analysis}

The JASPER model organizes permissions into patterns and relationships between patterns as an object-oriented approach to inherit permissions. This inheritance structure can be thought of as a tree, with resources forming the leaves and the actor/user being the root of the tree that inherits the permissions from the leaves.

For each policy specification, SPRT builds a bottom-up hash tree (see Figure 4) through a method similar to the Merkle tree construction $[23,24]$. The hash computation starts from the Resources and proceeds up to the Actors by calculating the hash value of each node as a combination of the information in the node itself and the hash values of all the mapped nodes below it. For instance, a Role's hash value is calculated based on its associated Tasks values, which depend on their containers and resources.

This technique has many inherent properties. First, the hash values define an equivalence measure between the nodes. For instance,

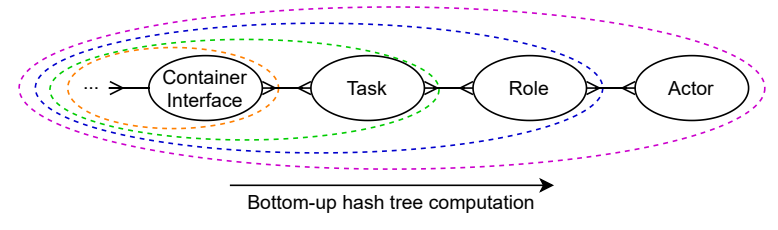

Figure 4: SPRT builds a bottom-up hash tree out of the JASPER AC model. The nested dotted ovals of different colors indicate that each node is considered for the hash value calculation of the mapped node(s) on the right.

if a Container Interface is the same in both policy specifications (e.g., no modifications in the mapped containers or resources), it has the same hash value. On the other hand, if changes are made, the hash value changes propagate from the Container Interface up to the Actors, which are implicitly connected. Then, once the hash trees are computed, SPRT performs a top-down hash value comparison starting from the tree's root (i.e., the Actors) and moving only to the tree branches where a change is detected, that is, where the hash values are different.

Figure 5 shows an example of how an RBAC policy change can be represented in SPRT. The dashed red line between the Git task and the Software Developer role denotes that the mapping was removed. Instead, the Git task is now mapped onto the Base role. The Software developer and the Researcher roles inherit the Base role's access permissions and thus can access the Git task. Since Alice and Bob are mapped to the Software developer and the Researcher roles, they can access the Git task. Ultimately, in the example, nothing changes for Alice in terms of the tasks she can access since she already had access to the Git task. The difference is that she gets the same access through the Role permission inheritance hierarchy. On the other hand, Bob obtains access to the Git task, a privilege he did not have before the policy change.

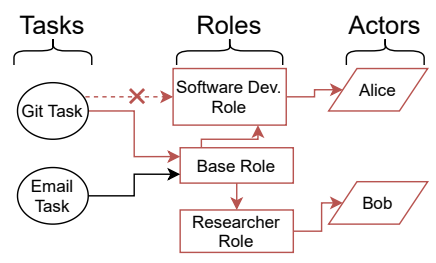

Figure 5: AC policy change example. The dashed red line indicates that the mapping was removed. The solid red color shows the Roles and Actors affected by that change.

\section{RESEARCH DIRECTIONS}

This section discusses research directions related to the approach outlined in the paper.

\subsection{Software Engineering Graph Policies}

At an abstract level working with graphs and linking nodes between graphs makes a clean and concise model. However, transitioning this approach to a real-world practice like software engineering faces several challenges. The communication requirements graph 
$C$ represents a common pattern, but as with software implementation patterns, there are many pattern variants, and selecting when to parameterize the pattern or make a new pattern becomes an engineering choice. Also, many of the requirements patterns are anchored by individual applications' requirements, which may change for new versions of the application. This creates the need for a complex pattern library that describes clear trade-offs in the various patterns and ties them to the versions implemented on the local network.

Another engineering consideration is the namespace of the nodes in each graph. Since each graph is meant to be completed by different individuals, they may choose different names for the semantically same node or give semantically different nodes the same name. Each graph having its own namespace is insufficient because various organizations will make communication requirements graphs. For example, when trying to map services to network hosts duplicate service names will potentially introduce errors. In the current Java implementation, the requirements graphs publish public strings defining the service, which the local administrator must assign to a host. In the end, it produces brittle code that needs a more robust way of addressing the naming of patterns and mapping them to concrete hosts.

\subsection{The Policy Rules Placement Problem}

The network compiler has choices on where to install rules to enforce the policy. For example, suppose the path between two nodes that should not communicate goes through two routers. The compiler could stop the communication on the first, second, or both routers. Placing the rule on multiple routers may increase security at the cost of network performance. Thus, it is critical to devise a systematic and automatic approach for rule placement able to ensure security and at the same time minimize overhead.

A naive network compiler may create a set of rules that allows necessary communications and stops all unneeded communications, and place this set on every PEP. Such a placement choice results in defense-in-depth ensuring policy enforcement if a PEP becomes compromised. However, network performance will fall as PEPs must evaluate more and more rules. For instance, consider a rule $r$ that involves two hosts $A$ and $B$ and a PEP that is not on any path between $A$ and $B$. Placing $r$ on that PEP has no impact on the packet filtering process but increases the rule matching time, since the PEP must compare each incoming packet against $r$ even though it is unlikely any packet will match $r$. Carefully selecting rule order could alleviate this performance penalty by putting rules unlikely to match at the end of the sequence of checks. The network compiler must also address PEPs that may be resource-constrained (e.g., can only enforce a limited number of rules) and packet transformation processes such as Network Address Translation (NAT) that hides IP addresses on certain sub-nets.

To enhance performance, one could leverage the network topology knowledge in the rule placement. A possible approach could be the following. First, it would be necessary to compute reachability for all nodes - a very well-known problem in graph theory for which there are algorithms with polynomial time complexity. Then, a rule should be placed only on PEPs that are on paths connecting the nodes involved in the rule. For instance, given a rule
$A \rightarrow B:$ deny, this rule should be placed only on PEPs on the paths that connect $A$ to $B$. This enhancement could improve the rule matching overhead by reducing the number of rules on each PEP while still providing resiliency in the scenario in which one or more PEPs are compromised. Such an approach has, however, two limitations: 1) it still entails significant rule redundancy on PEPs which are located on the same network's paths and might deploy large numbers of rules; 2) it does not leverage any network knowledge other than topology, such as, for example, the type of network PEPs and nodes that lie behind them or the type of traffic that flows through each network path. For instance, it is possible to distinguish between different classes (e.g., firewalls, middleboxes) of PEPs based on their security capabilities. Similarly, the security relevance of different nodes can vary, as a server providing a critical service or hosting a database of sensitive information is more security-sensitive than a user's laptop temporarily connected to the network. Thus, rule placement strategies leveraging this knowledge can substantially improve the network performances by enforcing security based on the network structure and minimizing the computational overhead of rule matching.

Towards this objectives, we outline two research directions:

(1) Metrics-based heuristics. The compiler should be able to evaluate each rule placement decision based on how it affects network security and overhead. Metrics-based heuristics can be used to address two different problems: rule placement decision making and rule placement evaluation.

(2) Machine learning (ML)-based techniques. One such technique is reinforcement learning based on reward/penalty functions that would encourage rules placement decisions based on efficiency.

Heuristics for rule placement decision making. They refer to heuristics to guide the compiler while generating and placing rules on the network's PEPs. As the policy compiler produces rules iteratively, it is necessary to define a method to place the newly generated rules to satisfy both communication and security requirements. Each rule addition impacts the network configuration; thus, the compiler should automatically evaluate the current rule placement and network topology to make informed decisions. One can achieve this by defining heuristics to evaluate each rule placement impact. However, as the aim is to improve the overall security while limiting the computational overhead, it is necessary to identify basic metrics that represent specific aspects of the network that are critical from a policy enforcement standpoint. For instance, below, we present four metrics that one could consider when defining the aforementioned heuristics:

- Strength: a function of the security capabilities associated with each PEP, which quantifies the PEP's reliability and resiliency to security attack.

- Worth: a function of each node's relevancy towards ensuring the network functionality. It is useful to identify critical sections of the network.

- Node impact the impact of placing a rule on a node $n$ as a function of the number of nodes affected by $n$. This metric measures the impact of a specific rule placement decision on the rest of the network. For instance, rules placed on a firewall that controls access to an entire private network 
impact more nodes than rules placed on a terminal router which only impact small sub-networks.

- cost: a function of the cost associated with each node based on the number of rules placed on that node. A high number of rules on one node can slow down the rule matching process on the examined packets and introduce undesired packet transmission delay.

In addition to metrics, it is necessary to define a formal model to combine them into one heuristic function to guide the compiler in the rule placement process. One could employ strength, worth, and node impact to model the network's overall security and the cost to represent the computational overhead due to rule matching. Hence, the heuristic function could provide the network compiler with the optimal rule placement decisions based on the placement's impact on security and cost. Identifying the basic metrics and combining them into a unified function is fundamental to enhance security and minimize overhead.

Heuristics for a rule placement evaluation. Heuristics are needed that enable a meaningful comparison between different rule placement configurations. For instance, consider a network administrator who manually alters the network policy to enhance the network security or change communication policies. In this case, a security policy testing tool like SPRT should describe how the changes affect the communication requirements, security and network performance. Such a comparison requires a metric function to evaluate the two rule placement configurations. The metric values should represent a combination of the features expected in the network to give the administrator information to determine which configuration provides better performance. As for defining metrics to guide the rule placement decision process, one can define the metrics to address this problem as the combination of different elements (e.g., strength, worth, node impact, and cost). However, in this case, the metrics do not help the network compiler decide where to place a newly generated rule but rather compare different rule placement configurations. As for the task discussed above, the challenge is defining the proper function combining the network's desired properties. Note that although one could think of using the same metrics for both tasks, they are slightly different and might require different approaches. In one case, it is necessary to guide the network compiler in placing each generated rule individually by finding the best fit for the current policy enforcement configuration. In the other case, the metrics provide a score representing the network's overall configuration and are used for comparison purposes.

Reinforcement learning guided rule placement. An alternative solution for the rule placement problem that does not require defining domain-based heuristics is to leverage ML techniques. Specifically, one could employ an approach based on reinforcement learning (RL) algorithms for the following reasons:

- RL does not require labeled input/output.

- $\mathrm{RL}$ is based on an agent that interacts with an environment by performing actions based on the environment's observations [19]. One could model the elements in our context as follows: the agent performs actions by pushing rules into PEPs, based on observations of the current rule placement configurations. At each step, the agent selects the optimal solution (i.e., selecting PEP(s) to push a rule) accordingly with a reward function.

- The reward function models the idea that the compiler should place a newly generated rule to improve the network's performance. For instance, more robust network security entails a higher reward, whereas high cost entails lower reward.

Although RL appears to be a compelling approach to address the rule placement problem, it requires addressing several issues. First, in most cases, it is necessary to model the environment as a Markov decision process ([19]). Second, the reward function should accurately reflect the impact of the agent's actions (push rules into PEPs) and guide the agent towards better decisions. The definition of the reward function requires further exploration of the domain space and identifying the elements that better represent the network performance.

Previous work. A problem similar to the optimal rule placement problem was addressed by Hamed et al. [16] first and Baiocchi et al. [4] later. In their research, the authors propose a framework to optimize policy rules ordering within network devices systematically, but they do not consider optimizing the rules across different devices. On the other hand, we outline a research direction that aims to optimize the rule placement on the entire network rather than on a single device. Nonetheless, a thorough approach for policy enforcement optimization could consider both the problems and combine the two objectives into one.

A different work, done by Rullo et al. [30], addresses the problem of allocating security resources on IoT networks. The authors present a method to automatically compute the optimal security resource allocation strategy that minimizes the total energy consumption, the installation cost, and the network's criticality. Similar to the research direction we outline, the problem is to optimize the allocation strategy according to multiple objectives. However, Rullo et al. focus on security tools allocation rather than network policy rules. One could combine the two ideas first to allocate PEPs on the network and subsequently place the rules implementing the required network policies on the optimal PEPs.

\subsection{Path Validation}

Path validation in networks is a critical task for security and reliability of the networks [9]. Path validation is the combination of two main sub-tasks: path enforcement and path verification [9]. For the former, a source node should be able to specify the path (or paths) that a packet must follow to reach a destination node, and the intermediate hops on the path should be able to enforce the path when processing the packet. For the latter, both the destination node and the intermediate nodes should be able to verify whether a packet is following the pre-determined path or not. Regardless of the techniques used for path enforcement and validation, one common drawback is that these techniques introduce significant overhead on both communication and processing. Every packet has to carry additional fields to keep track of the specified path and the traversed nodes. Each node in the path must verify the content of such fields to guarantee that the packet is flowing through the specified path and discard it when detecting irregularities. Moreover, the introduction of additional fields requires modifying the routing protocols. 
Since the network compiler employs a graph-based representation to model the network requirements, one can represent path enforcement requirements as an additional graph in the graphs set given as the compiler's input. As the compiler also has complete knowledge of the network topology and control over the node's configuration, we believe it is possible to enable path enforcement through network policies. In other words, one could leverage the network compiler's capabilities to produce rules that enforce specific paths automatically and to optimally place network PEPs to enforce and validate paths so as to minimize overhead.

\subsection{Negative Constraint Explosion}

The communication requirements graph allows us to mathematically define the set of negative constraints of denied network flows. Figure 3 shows all the negative constraints for the communication requirements graph portion in Figure 2. Even in this small example, the number of denied constraints is much larger than the allowed constraints. For larger networks, the number of denied constraints will grow exponentially.

In previous experiments, we tried using an SMT-based program synthesizer to generate router policies across a network. This approach used a small language inspired by Berkeley Packet Filter [21] as an abstraction around iptables and used the requirements graph to generate both the positive and negative constraints that the network policy must satisfy. The approach worked on small examples, but the exponential growth of denied constraints caused a corresponding explosion in the run time of the solver.

Approaches that reduce the number of negative constraints based on network topology may sufficiently reduce the problem's size. For example, if all the communication goes through a choke point like a firewall, defining specific rules on the firewall could significantly reduce the number of negative constraints to check. Future research could also look at other ways of breaking the network up into subgraphs, analyze the smaller sub-graph and then compose the results to make security statements about the entire network.

\subsection{Capturing Communication Requirements}

Our approach requires the communication requirements graph $C$, which represents knowledge most organizations do not possess. As the number of services grows, the number of supporting services and interrelationships also grows. Often even application developers are not aware of the transitive closure of all the services their application depends on. Tools are needed that either extract dependencies from the source code or infer the dependencies by monitoring applications operating on live networks.

Analyzing the application source code to locate the system calls to the network interfaces and extracting details about the required communication faces a number of challenges. The code often binds with specific services using instance data like IP addresses, making extracting communication patterns difficult. Moreover, the common pattern of delegating work to supporting libraries or other agents will further obscure dependencies.

Capturing the dependencies from monitoring network traffic also poses significant challenges. High volumes of traffic and multiple services running concurrently will obscure the dependencies, and the approach will also have to map network traffic to the process running on the host responsible for the traffic. However, one could discover the core dependency relationships by running services in a less noisy test environment.

A hybrid approach that combines both types of analysis with application development seems like the most promising avenue. A SPRT-like tool using a more realistic network model with simulated PEPs could be used to validate the resulting communications requirements graph. The graph could also be independently vetted and adjusted by the community of developers to improve it over time, much like long-term software development.

Techniques have been proposed for capturing database accesses by applications $[8,12]$. Such techniques could be extended for capturing network transaction activities.

\subsection{Software-Defined Networks}

The definition of the network compiler part of our framework is closely related to software-defined networks (SDN) [5]. SDN typically distinguish between two planes: the control and data (i.e., forwarding) planes. The control plane implements the network's logic, including access control, into modules called controllers. In contrast, the forwarding plane is the translation of this logic into forwarding decisions sent to network devices, commonly referred to as switches, using a protocol such as OpenFlow [22]. The controller's operation essentially resembles that of the network compiler. Architectures inspired from SDN such as Ethane (Casado et al. [10]) and Resonance (Nayak et al. [26]) could extend our graph-based approach by deploying the least privilege communications policy on the network's controllers. Furthermore, SPRT can test the network access control policy and expose unnecessary flows, thus narrowing the attack surface. SPRT could also be used to reason about policy changes performed at the control plane.

\subsection{Firewall Ruleset Analysis}

The problem of firewall misconfigurations has been already addressed [17] by proposing algorithms $[1,2,11]$ or automatic tools [7, $15,27]$ to detect and remove configuration errors. Despite their effectiveness in finding discrepancies in the deployed rules (e.g., rule shadowing and redundancy), such approaches lack higher-level semantic reasoning, for example, whether the rules are relevant and accurately reflect higher-level policies. The use of our framework could enhance such previous approaches.

\subsection{Industrial Control Systems}

Bertolotti et al. [6] propose a twofold model for analyzing rolebased access control policies in Industrial Network Systems (INS). They model an INS through two views: the specification view and the implementation view. Specification views capture an RBAC model, whereas the implementation view captures the underlying network topology and the spatial location of physical objects that are relevant for security (i.e., rooms, gates, cabinets, and so on). The goal of the approach is to determine whether the RBAC model expressed by the specification view reflects the policy enforcement expressed by the implementation view. However, their analysis does not consider the network configuration (i.e., the traffic control rules on firewalls, switches, or routers). Furthermore, the approach focuses on understanding users' permissions on resources and does 
not consider the interaction between resources. Our framework, if integrated with their approach, would provide means to evaluate different network aspects and define more precisely network access control for INS systems.

\section{CONCLUSION}

This paper proposes a novel graph-based policy specification framework to capture semantics and access control patterns for a coherent understanding of the network and its requirements. We use the very same semantics for both policy compilation [34] and testing. Testing is critical to determine whether the deployed network policies enable applications and users to perform their missions successfully while ensuring a least privilege policy. We have designed and implemented a Security Policy Regression Testing (SPRT) tool to support network-aware policy analysis and verification. As policy changes may happen frequently and their impact may not always be apparent to the policy administrators, SPRT also performs policy change impact analysis; it compares the policy specifications and identifies what has changed and the impact of those changes.

Our work opens several research directions. Our communication requirements graph has an inherent capability to specify and enforce communication paths; an example is that all guests' traffic should pass through an intrusion detection software running on a middlebox. It would also be interesting to investigate the use of our framework in conjunction with path validation protocols. Our framework could also be extended and applied to other domains, such as software-defined networks and industrial control systems.

\section{ACKNOWLEDGMENTS}

This research was supported by the Air Force Research Laboratory, Rome, NY, under contract number FA8750-19-C-0082.

\section{REFERENCES}

[1] Ryma Abassi and Sihem Guemara El Fatmi. 2008. An Automated Validation Method for Security Policies: the firewall case. In 2008 The Fourth International Conference on Information Assurance and Security. IEEE, 291-294.

[2] Joaquin Garcia Alfaro, Nora Boulahia-Cuppens, and Frédéric Cuppens. 2008 Complete analysis of configuration rules to guarantee reliable network security policies. International fournal of Information Security 7, 2 (2008), 103-122.

[3] Fabien Autrel, Frédéric Cuppens, N Cuppens-Boulahia, and Celine Coma. 2008 MotOrBAC 2: a security policy tool. In 3rd Conference on Security in Network Architectures and Information Systems (SAR-SSI 2008), Loctudy, France. 273-288.

[4] A. Baiocchi, G. Maiolini, Annachiara Mingo, and D. Goretti. 2015. Adaptive Conflict-Free Optimization of Rule Sets for Network Security Packet Filtering Devices. 7. Comput. Networks Commun. 2015 (2015), 872326:1-872326:17.

[5] Kamal Benzekki, Abdeslam El Fergougui, and Abdelbaki Elbelrhiti Elalaoui. 2016. Software-defined networking (SDN): a survey. Security and communication networks 9, 18 (2016), 5803-5833.

[6] Ivan Cibrario Bertolotti, Luca Durante, Lucia Seno, and Adriano Valenzano. 2015 A twofold model for the analysis of access control policies in industrial networked systems. Computer Standards \& Interfaces 42 (2015), 171-181.

[7] Chiara Bodei, Pierpaolo Degano, Letterio Galletta, Riccardo Focardi, Mauro Tempesta, and Lorenzo Veronese. 2018. Language-independent synthesis of firewall policies. In 2018 IEEE European Symposium on Security and Privacy (EuroS\&P). IEEE, 92-106

[8] Lorenzo Bossi, Elisa Bertino, and Syed Rafiul Hussain. 2016. A system for profiling and monitoring database access patterns by application programs for anomaly detection. IEEE Transactions on software engineering 43, 5 (2016), 415-431.

[9] Kai Bu, Avery Laird, Yutian Yang, Linfeng Cheng, Jiaqing Luo, Yingjiu Li, and Kui Ren. 2020. Unveiling the Mystery of Internet Packet Forwarding: A Survey of Network Path Validation. ACM Comput. Surv. 53, 5, Article 104 (Sept. 2020), 34 pages. https://doi.org/10.1145/3409796

[10] Martin Casado, Michael J Freedman, Justin Pettit, Jianying Luo, Nick McKeown, and Scott Shenker. 2007. Ethane: Taking control of the enterprise. ACM SIGCOMM computer communication review 37,4 (2007), 1-12.
[11] Frédéric Cuppens, Nora Cuppens-Boulahia, and Joaquin Garcia-Alfaro. 2019. Misconfiguration management of network security components. arXiv preprint arXiv:1912.07283 (2019).

[12] Daren Fadolalkarim, Elisa Bertino, and Asmaa Sallam. 2020. An Anomaly Detection System for the Protection of Relational Database Systems against Data Leakage by Application Programs. In 2020 IEEE 36th International Conference on Data Engineering (ICDE). IEEE, 265-276.

[13] David F Ferraiolo, Ravi Sandhu, Serban Gavrila, D Richard Kuhn, and Ramaswamy Chandramouli. 2001. Proposed NIST standard for role-based access control. ACM Transactions on Information and System Security (TISSEC) 4, 3 (2001), 224-274.

[14] Kathi Fisler, Shriram Krishnamurthi, Leo A Meyerovich, and Michael Carl Tschantz. 2005. Verification and change-impact analysis of access-control policies. In Proceedings of the 27th international conference on Software engineering. 196-205.

[15] Joaquin Garcia-Alfaro, Frédéric Cuppens, Nora Cuppens-Boulahia, and Stere Preda. 2010. MIRAGE: a management tool for the analysis and deployment of network security policies. In Data Privacy Management and Autonomous Spontaneous Security. Springer, 203-215.

[16] Hazem Hamed and Ehab Al-Shaer. 2006. Dynamic Rule-Ordering Optimization for High-Speed Firewall Filtering. In Proceedings of the 2006 ACM Symposium on Information, Computer and Communications Security (Taipei, Taiwan) (ASIACCS '06). Association for Computing Machinery, New York, NY, USA, 332-342. https: //doi.org/10.1145/1128817.1128867

[17] Hazem Hamed and Ehab Al-Shaer. 2006. Taxonomy of conflicts in network security policies. IEEE Communications Magazine 44, 3 (2006), 134-141.

[18] Vincent C Hu, Rick Kuhn, and Dylan Yaga. 2017. Verification and test methods for access control policies/models. NIST Special Publication 800 (2017), 192.

[19] L. P. Kaelbling, M. L. Littman, and A. W. Moore. 1996. Reinforcement Learning: A Survey. Fournal of Artificial Intelligence Research (JAIR) (1 May 1996). https: //www.jair.org/index.php/jair/article/view/10166

[20] Brian Krisler, Partha Pal, Zech Bertilson, and Dan Thomsen. 2019. Secure Desktop Computing in the Cloud. In 2019 6th IEEE International Conference on Cyber Security and Cloud Computing (CSCloud)/2019 5th IEEE International Conference on Edge Computing and Scalable Cloud (EdgeCom). IEEE, 107-112.

[21] Steven McCanne and Van Jacobson. 1993. The BSD Packet Filter: A New Architecture for User-level Packet Capture.. In USENIX winter, Vol. 46.

[22] Nick McKeown, Tom Anderson, Hari Balakrishnan, Guru Parulkar, Larry Peterson, Jennifer Rexford, Scott Shenker, and Jonathan Turner. 2008. OpenFlow: enabling innovation in campus networks. ACM SIGCOMM computer communication review 38, 2 (2008), 69-74.

[23] Ralph Merkle. 1979. Merkle tree patent.

[24] Ralph C Merkle. 1987. A digital signature based on a conventional encryption function. In Conference on the theory and application of cryptographic techniques. Springer, 369-378.

[25] John Moy et al. 1998. OSPF version 2. (1998)

[26] Ankur Kumar Nayak, Alex Reimers, Nick Feamster, and Russ Clark. 2009. Resonance: Dynamic access control for enterprise networks. In Proceedings of the 1st ACM workshop on Research on enterprise networking. 11-18.

[27] Timothy Nelson, Christopher Barratt, Daniel J Dougherty, Kathi Fisler, and Shriram Krishnamurthi. 2010. The Margrave Tool for Firewall Analysis.. In LISA. $1-18$.

[28] Palo Alto Networks. [n.d.]. What is a Zero Trust Architecture. https://www. paloaltonetworks.com/cyberpedia/what-is-a-zero-trust-architecture

[29] Scott Rose, Oliver Borchert, Stu Mitchell, and Sean Connelly. 2019. Zero trust architecture. Technical Report. National Institute of Standards and Technology.

[30] Antonino Rullo, Daniele Midi, Edoardo Serra, and Elisa Bertino. 2017. Pareto Optimal Security Resource Allocation for Internet of Things. ACM Trans. Priv. Secur. 20, 4, Article 15 (Oct. 2017), 30 pages. https://doi.org/10.1145/3139293

[31] Donnie Savage, James Ng, Steven Moore, Donald Slice, Peter Paluch, and Russ White. 2016. Cisco's enhanced interior gateway routing protocol (eigrp). In RFC Editor.

[32] Dan Thomsen. 2007. Patterns in Security Enforcement Policy Development. In 18th International Workshop on Database and Expert Systems Applications (DEXA 2007). IEEE, 744-748.

[33] Dan Thomsen. 2011. Practical policy patterns. In Proceedings of the first ACM conference on Data and application security and privacy. 225-230.

[34] Dan Thomsen and Elisa Bertino. 2018. Network policy enforcement using transactions: The neutron approach. In Proceedings of the 23nd ACM on Symposium on Access Control Models and Technologies. ACM, 129-136.

[35] Aristotelis Tsirigos and Zygmunt J Haas. 2004. Analysis of multipath routingPart I: The effect on the packet delivery ratio. IEEE Transactions on wireless communications 3,1 (2004), 138-146. 